\title{
Seeding rate and cultivar impacts on nutrient uptake of field pea under fertile soil condition
}

\author{
Baris Bülent Asik ${ }^{1 *}$, Aysen Uzun ${ }^{1}$, and Esvet Acikgöz ${ }^{1}$ \\ 'Bursa Uludag University, Faculty of Agriculture, Gorukle Campus, 16059, Bursa, Turkey. \\ "Corresponding author (bbasik@uludag.edu.tr).
}

Received: 8 July 2019; Accepted: 1 October 2019; doi:10.4067/S0718-58392020000100011

\begin{abstract}
Plant density is an important agronomic factor that affects crop growth, development, and yield. The optimum plant density to attain the highest yield can vary with genotype, production and, environmental factors. A field experiment was conducted at Bursa Uludag University Agricultural Area to evaluate the effects of seeding rate and variety on plant nutrient uptake of field pea (Pisum sativum L.) cultivars (Ulubatli, Kirazli, Golyazi, and Urunlu). Some very important factors responsible for low yield are plant density under field conditions and the selection of unsuitable cultivars. The adoption of pea cultivars that are more productive and better adapted to cultivation regions and the increase in plant population per area are important strategies to obtain higher productivity under field conditions. Plant density is one of the most effective agronomic factors for determining optimum plant nutrient uptake. The factorial experiment was arranged in a completely randomized block design with three replicates per treatment. Four cultivars and five seeding rates (75,

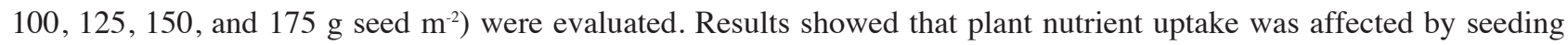
rate and pea cultivars. The maximum rate of plant nutrient uptake occurred at a density of 100-125 $\mathrm{g} \mathrm{seed} \mathrm{m}^{-2}$ and for 'Kirazli', 'Golyazi', and 'Urunlu'. However, 'Ulubatli' showed minimum values of nutrient uptake. Regarding macroand micronutrients, varieties on the average had mean nutrient uptake with the following sequence: $\mathrm{N}>\mathrm{K}>\mathrm{Ca}>\mathrm{P}>\mathrm{Mg}$, $\mathrm{Na}, \mathrm{Fe}>\mathrm{Mn}>\mathrm{Zn}>\mathrm{Cu}$. Pea varieties and seeding rates should be considered in the fertilization program.
\end{abstract}

Key words: Cultivars, pea, Pisum sativum, plant nutrient, seeding rates.

\section{INTRODUCTION}

Plant density is one of the most effective agronomic factors for determining yield, which is individually affected by cultivars and climatic conditions as well as the production system (Zandi et al., 2011). Optimum crop plant density varies considerably depending on the conditions of the growing area and soil fertility status. Plant distance is an important factor for higher production and gives plants an equal opportunity to survive and make the best use of other inputs (e.g., water, plant nutrients). Many studies have been conducted with the aim of determining the optimum plant density or seeding rate (Cupina et al., 2010; Dahmardeh et al., 2010; Thandiwe and Rickertsen, 2011; Türk et al., 2011; Uzun et al., 2017).

When planting density is relatively low, resources (water, light, and plant nutrients) are made available for plant growth, and competition for these resources is relatively low. As planting density increases, competition also increases, and plant growth tends to decrease. There is therefore an optimal planting density, that is, less than optimal densities favor growth and greater than optimal densities reduce growth (Siqueira et al., 2014). For each crop and under specific environmental conditions, the optimum plant population should usually be determined by local research.

Peas (Pisum sativum L.) are widely grown for hay, pasture, or silage production. Field pea ( . sativum) is an important crop for its protein content (Olle, 2017). The most widely grown pea varieties in Turkey are "Ulubatli, Kirazli, Golyazi, and Urunlu". As a forage crop, pea hay and seed have high-quality protein, are rich in $\mathrm{P}$ and $\mathrm{Ca}$, and are a good source 
of vitamins, especially vitamins A and $\mathrm{D}$. These qualities make field pea one of the best animal feeds and is almost indispensable for efficient and economical livestock feeding (Tan et al., 2012). A wide range of field pea varieties are currently being evaluated for adaptation and yield performance. In addition, fertilization recommendations for pea production in the growing areas must be developed (Türk et al., 2011). The objectives of this research study were to study the effect of different seeding rates and cultivars on plant nutrient uptake in four field pea cultivars under fertility soil conditions. The amount of fertilizer can therefore be determined on the basis of planting density and economic benefits.

\section{MATERIALS AND METHODS}

\section{Experimental area}

Field trials were conducted in 2010 and 2011 in the experimental area of the Department of Field Crops, Faculty of Agriculture, Bursa Uludag University located in the Bursa Province, Marmara Region, Turkey. The experimental fields were located in the coastal area of northwest Turkey $\left(40^{\circ} 13^{\prime} \mathrm{N}, 28^{\circ} 52^{\prime} \mathrm{E}\right.$; 70 m.a.s.l.) The mean temperatures recorded by the State Meteorology Department during the plant growth period (November to June) in the trial area are shown in Table 1.

\section{Field experiment}

The factorial experiment was arranged in a completely randomized block design with three replicates per treatment. Four pea (Pisum sativum L.) cultivars (Kirazli, Ulubatli, Golyazi, and Urunlu) and five sowing rates $(75,100,125,150$, and $175 \mathrm{~g}$ seed $\mathrm{m}^{-2}$ ) were evaluated. Two semi-leafless cultivars (Ulubatli and Kirazli) and two leafy cultivars (Golyazi and Urunlu) were used in this study. Field pea cultivars were sown with an Oyjort experimental drill. The plot size was 1.4 $\times 10=14 \mathrm{~m}^{2}$, which consisted of 8 rows with $17.5 \mathrm{~cm}$ spacing. The previous crop was wheat (Triticum sativum Lam.) in 2010 and 2011. Before seeding, $30 \mathrm{~kg} \mathrm{~N} \mathrm{ha}^{-1}$ as ammonium nitrate (AN) was applied. Weeds were manually controlled as needed. No irrigation was applied during the growing season.

The soil is Eutric Vertisol according to the FAO/Unesco (1974 and 1990 classification system) (Özsoy and Aksoy, 2004; 2007). The soil is clay loam, which is deep and slightly alkaline (pH 7.79 to 8.03 ), with a low calcium carbonate content $(1.61 \%$ to $2.67 \%)$ and a low organic matter content $(1.35 \%$ to $1.82 \%)$. The total $\mathrm{N}$ content per plot and year fluctuated between $0.098 \%$ and $0.127 \%, 31.85$ and $62.03 \mathrm{mg} \mathrm{P} \mathrm{kg}^{-1}$, and 1.25 and $0.68 \mathrm{meq} \mathrm{K} 100 \mathrm{~g} \mathrm{~g}^{-1}$. Soil micronutrient content was sufficient for pea production (Table 2) (Kacar, 2014). Before sowing, $\mathrm{N}\left(30 \mathrm{~kg} \mathrm{~N} \mathrm{ha}^{-1}\right), \mathrm{P}\left(30 \mathrm{~kg} \mathrm{P}_{2} \mathrm{O}_{5} \mathrm{ha}^{-1}\right)$, and $\mathrm{K}\left(30 \mathrm{~kg} \mathrm{~K}_{2} \mathrm{O} \mathrm{ha}{ }^{-1}\right), 15: 15: 15$, were applied to the plots as basal fertilizer.

Plant analysis. At harvest time, all the plants of each treatment within $1 \mathrm{~m}^{2}$ were harvested and a number of samples were taken to determine plant nutrient concentrations. Plant samples were rinsed with tap water and rinsed twice with deionized water. Plant material was dried in a forced-air oven at $65^{\circ} \mathrm{C}$ for $48 \mathrm{~h}$. Plant dry weights were determined and plot yield was calculated. Dried plants were used in the analysis. Results were evaluated according to plot DM yield. Plant total N was determined with a Buchi K-437/K-350 digestion/distillation unit (BUCHI Labortechnik AG, Flawil, Switzerland) according to the Kjeldahl method. Total elements of plant samples were digested with $\mathrm{HNO}_{3}$ and $\mathrm{H}_{2} \mathrm{O}_{2}$ (Berghof MWS 2

Table 1. Temperature, precipitation, and relative humidity values for experimental years and long-term growing seasons in Bursa, Turkey.

\begin{tabular}{|c|c|c|c|c|c|c|c|c|c|}
\hline \multirow[b]{2}{*}{ Month } & \multicolumn{3}{|c|}{ Temperature } & \multicolumn{3}{|c|}{ Precipitation } & \multicolumn{3}{|c|}{ Relative humidity } \\
\hline & 2010 & 2011 & Long-term & 2010 & 2011 & Long-term & 2010 & 2011 & Long-term \\
\hline & 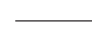 & $-{ }^{\circ} \mathrm{C}$ & 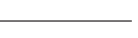 & 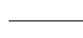 & $-\mathrm{mm}$ & - & & $\%$ & 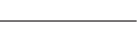 \\
\hline November & 10.0 & 15.5 & 10.3 & 80.6 & 24.0 & 85.4 & 84.5 & 68.6 & 72.4 \\
\hline December & 9.8 & 9.5 & 7.1 & 119.1 & 152.6 & 96.4 & 77.7 & 79.8 & 71.7 \\
\hline January & 6.6 & 5.8 & 5.4 & 149.7 & 72.4 & 80.3 & 77.3 & 81.5 & 71.2 \\
\hline February & 9.4 & 6.1 & 5.9 & 178.9 & 18.4 & 66.2 & 77.4 & 74.4 & 69.6 \\
\hline March & 9.0 & 8.2 & 8.5 & 115.3 & 67.4 & 62.7 & 77.8 & 77.0 & 68.9 \\
\hline April & 13.5 & 10.6 & 13.0 & 63.4 & 76.8 & 65.2 & 71.3 & 78.3 & 67.1 \\
\hline May & 19.3 & 16.8 & 17.7 & 29.4 & 27.3 & 43.4 & 64.3 & 75.7 & 64.8 \\
\hline June & 22.7 & 22.2 & 22.4 & 135.2 & 14.0 & 33.6 & 68.8 & 63.3 & 58.7 \\
\hline Total/Mean & 12.5 & 11.8 & 11.3 & 871.6 & 452.9 & 533.2 & 74.9 & 74.8 & 68.1 \\
\hline
\end{tabular}


Table 2. Selected soil properties.

\begin{tabular}{|c|c|c|c|c|}
\hline Properties & 2010 & Classes & 2011 & Classes \\
\hline Sand, $\%$ & 33.9 & & 33.9 & \\
\hline Silt, \% & 19.7 & Clay loam & 17.6 & Clay loam \\
\hline Clay, \% & 46.6 & & 48.5 & \\
\hline $\mathrm{pH}$ & 8.03 & Alkaline & 7.79 & Slightly alkaline \\
\hline Electrical conductivity, $\mu \mathrm{S} \mathrm{cm}^{-1}$ & 652.0 & Saltless & 390.0 & Saltless \\
\hline Lime, \% & 2.67 & Low & 1.61 & Low \\
\hline Organic matter, $\%$ & 1.82 & Low & 1.35 & Low \\
\hline Total N, \% & 0.127 & Sufficient & 0.098 & Sufficient \\
\hline $\mathrm{NH}_{4}-\mathrm{N}, \mathrm{mg} \mathrm{kg}^{-1}$ & 92.86 & & 45.45 & \\
\hline $\mathrm{NO}_{3}-\mathrm{N}, \mathrm{mg} \mathrm{kg}^{-1}$ & 22.10 & & trace & \\
\hline Available P, $\mathrm{mg} \mathrm{kg}^{-1}$ & 62.03 & Excessive & 31.85 & Excessive \\
\hline Available $\mathrm{K}, \mathrm{cmol} \mathrm{kg}^{-1}$ & 1.246 & Excessive & 0.685 & Sufficient \\
\hline Available $\mathrm{Na}$, cmol kg-1 & 0.504 & Sufficient & 0.361 & Sufficient \\
\hline Available Ca, $\mathrm{cmol} \mathrm{kg}^{-1}$ & 55.88 & Excessive & 34.93 & Excessive \\
\hline DTPA extractable $\mathrm{Cu}, \mathrm{mg} \mathrm{kg}^{-1}$ & 2.000 & Sufficient & 1.937 & Sufficient \\
\hline DTPA extractable $\mathrm{Zn}, \mathrm{mg} \mathrm{kg}^{-1}$ & 0.885 & Sufficient & 0.509 & Sufficient \\
\hline DTPA extractable $\mathrm{Mn}, \mathrm{mg} \mathrm{kg}^{-1}$ & 29.81 & Sufficient & 31.95 & Sufficient \\
\hline DTPA extractable Fe, $\mathrm{mg} \mathrm{kg}^{-1}$ & 6.382 & Excessive & 11.88 & Excessive \\
\hline
\end{tabular}

DTPA: Diethylenetriaminepentaacetic acid.

DAP 60K, Berghof Products+Instruments, Eningen, Germany), P was measured colorimetrically, and the other elements $(\mathrm{K}, \mathrm{Ca}, \mathrm{Mg}, \mathrm{Fe}, \mathrm{Cu}, \mathrm{Zn}, \mathrm{Mn}$ ) were analyzed from extracts by inductively coupled plasma-optical emission spectrometry (ICP OES) (OPTIMA 2100 DV; Perkin Elmer, Waltham, Massachusetts, USA) (Kacar, 2014).

An ANOVA was performed with the JMP 5.0.1 software (1989-2002; SAS Institute, Cary, North Carolina, USA). The statistical significance of the treatments was determined at the 0.05 and 0.01 probability levels by the F-test. For the differentiation of means, Duncan's test was applied.

\section{RESULTS AND DISCUSSION}

In the present study, the effects of seeding rate on plant nutrient ( $\mathrm{N}, \mathrm{P}, \mathrm{K}, \mathrm{Ca}, \mathrm{Mg}, \mathrm{Na}, \mathrm{Fe}, \mathrm{Cu}, \mathrm{Zn}$, and $\mathrm{Mn}$ ) uptake in four forage pea cultivars were determined. The ANOVA indicated that cultivars, seeding rates, and the cultivar $\times$ seeding rate, cultivar $\times$ year, and seeding rate $\times$ year interactions were significantly affected by nutrient uptake in the two experimental years (Table 3).

Plant nutrient uptake was calculated as the product of DM yield and plant nutrient concentrations (Table 4) (Uzun et al., 2017). According to our results, the highest DM yield was obtained for 'Kirazli' in both 2010 and 2011. Dry matter yields ranged from 0.749 to $0.979 \mathrm{~kg} \mathrm{~m}^{-2}$ for 'Kirazli' in both years. In the first $\left(0.795 \mathrm{~kg} \mathrm{~m}^{-2}\right)$ and second year

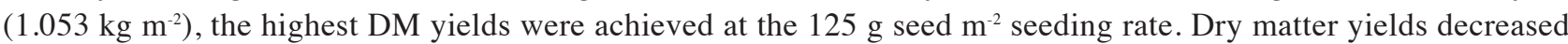
as seeding rates increased. Lateral branch and shoot number decreased with increasing seeding rates (especially at 150-175 $\mathrm{g} \mathrm{seed}^{-2}$ ) in both years. In the cultivar $\times$ seeding rate interaction, the highest DM yield was for 'Kirazli' at 125 seed $\mathrm{g} \mathrm{m}^{-2}$ with $0.932 \mathrm{~kg} \mathrm{~m}^{-2}$ in 2010 and $1.550 \mathrm{~kg} \mathrm{~m}^{-2}$ in 2011. Dry matter yield was higher in 2011 than in 2010.

Table 3. The ANOVA results for different plant variety traits at different seeding rates.

\begin{tabular}{|c|c|c|c|c|c|c|c|c|c|c|c|c|}
\hline Source & DF & $\begin{array}{c}\text { Dry } \\
\text { yield }\end{array}$ & $\mathrm{N}$ & $\mathrm{P}$ & $\mathrm{K}$ & $\mathrm{Ca}$ & $\mathrm{Mg}$ & $\mathrm{Na}$ & $\mathrm{Fe}$ & $\mathrm{Cu}$ & $\mathrm{Zn}$ & $\mathrm{Mn}$ \\
\hline Cultivar (C) & 3 & $*$ & $* *$ & $* *$ & $* *$ & $* *$ & $* *$ & $* *$ & $*$ & $* *$ & $* *$ & $* *$ \\
\hline Seeding rate $(\mathrm{D})$ & 4 & $*$ & $* *$ & $* *$ & $* *$ & $* *$ & $* *$ & $* *$ & $* *$ & $* *$ & $* *$ & $* *$ \\
\hline Year $(Y)$ & 1 & $*$ & $* *$ & $* *$ & $* *$ & $* *$ & $* *$ & $* *$ & $*$ & $* *$ & $* *$ & ns \\
\hline Cultivar $\times$ Seeding rate & 12 & $*$ & $* *$ & ns & ns & $* *$ & $* *$ & $* *$ & $* *$ & $*$ & ns & ns \\
\hline Cultivar $\times$ Year & 3 & $*$ & ns & ns & ns & ns & $*$ & ns & $* *$ & $* *$ & ns & $*$ \\
\hline Seeding rate $\times$ Year & 4 & $*$ & $* *$ & $*$ & ns & ns & $*$ & ns & $* *$ & ns & ns & ns \\
\hline $\mathrm{C} \times \mathrm{D} \times \mathrm{Y}$ & 12 & $*$ & $* *$ & ns & ns & ns & ns & ns & $* *$ & ns & ns & ns \\
\hline Error & 78 & & & & & & & & & & & \\
\hline
\end{tabular}

*,**Significant at the 0.05 and 0.01 probability levels, respectively; ns: nonsignificant; DF: degrees of freedom. 
Table 4. Effects of different seeding rates on dry matter yield measured in different cultivars.

\begin{tabular}{lllllll}
\hline \multicolumn{7}{c}{ DM yield, $\mathrm{kg} \mathrm{m}^{2}$} \\
\hline Seeding rate & $75 \mathrm{~g}$ & $100 \mathrm{~g}$ & $125 \mathrm{~g}$ & $150 \mathrm{~g}$ & $175 \mathrm{~g}$ & Mean \\
\hline Cultivars & & & 2010 & & \\
\hline Kirazli & $0.726 \mathrm{c}$ & $0.826 \mathrm{~b}$ & $0.932 \mathrm{a}$ & $0.675 \mathrm{de}$ & $0.588 \mathrm{~h}$ & $0.749 \mathrm{~A}$ \\
Ulubatli & $0.524 \mathrm{ij}$ & $0.666 \mathrm{ef}$ & $0.667 \mathrm{~d}-\mathrm{f}$ & $0.548 \mathrm{i}$ & $0.464 \mathrm{k}$ & $0.574 \mathrm{D}$ \\
Golyazi & $0.694 \mathrm{~d}$ & $0.731 \mathrm{c}$ & $0.845 \mathrm{~b}$ & $0.645 \mathrm{fg}$ & $0.519 \mathrm{j}$ & $0.687 \mathrm{~B}$ \\
Urunlu & $0.586 \mathrm{~h}$ & $0.636 \mathrm{~g}$ & $0.734 \mathrm{c}$ & $0.586 \mathrm{~h}$ & $0.458 \mathrm{k}$ & $0.600 \mathrm{C}$ \\
Mean & $0.633 \mathrm{C}$ & $0.715 \mathrm{~B}$ & $0.795 \mathrm{~A}$ & $0.613 \mathrm{D}$ & $0.507 \mathrm{E}$ & \\
\hline & & & & & \\
\hline Kirazli & $0.850 \mathrm{e}-\mathrm{g}$ & $1.076 \mathrm{~b}$ & $1.550 \mathrm{a}$ & $0.997 \mathrm{c}$ & $0.824 \mathrm{fg}$ & $0.979 \mathrm{~A}$ \\
Ulubatli & $0.671 \mathrm{j}$ & $0.846 \mathrm{e}-\mathrm{g}$ & $1.009 \mathrm{bc}$ & $0.867 \mathrm{e}-\mathrm{g}$ & $0.733 \mathrm{~h}-\mathrm{j}$ & $0.826 \mathrm{C}$ \\
Golyazi & $0.799 \mathrm{gh}$ & $0.984 \mathrm{~cd}$ & $1.041 \mathrm{bc}$ & $0.886 \mathrm{ef}$ & $0.802 \mathrm{gh}$ & $0.907 \mathrm{~B}$ \\
Urunlu & $0.715 \mathrm{ij}$ & $0.914 \mathrm{de}$ & $1.009 \mathrm{bc}$ & $0.743 \mathrm{hi}$ & $0.572 \mathrm{k}$ & $0.791 \mathrm{D}$ \\
Mean & $0.759 \mathrm{D}$ & $0.955 \mathrm{~B}$ & $1.053 \mathrm{~A}$ & $0.873 \mathrm{C}$ & $0.733 \mathrm{D}$ & \\
\hline
\end{tabular}

Uppercase letters in the same row and lowercase letters in the same column indicate differences $(P<0.05)$ according to Duncan's test.

Combined data demonstrated that the interaction between cultivar and plant density had significant effects on plant nutrient uptake. There were significant differences among pea cultivars; 'Kirazli' and 'Urunlu' had the highest value for $\mathrm{N}, \mathrm{P}, \mathrm{Ca}, \mathrm{Mg}, \mathrm{Na}, \mathrm{Fe}$, and $\mathrm{Cu}$ uptake. In addition, the highest value was obtained for the $125 \mathrm{seed} \mathrm{g} \mathrm{m}^{-2}$ treatment. The lowest value was recorded for 'Ulubatli' at the lowest plant density $\left(75 \mathrm{seed} \mathrm{g} \mathrm{m}^{-2}\right)$. These results demonstrate that the performance of the pea cultivars can vary according to plant density. This result has been previously described (Gan et al., 2003; Spies et al., 2010; Türk et al., 2011). Two semi-leafless cultivars (Ulubatli and Kirazli) and two leafy cultivars (Golyazi and Urunlu) were used in this study. Semere and Froud-Williams (2001) reported that semi-leafless field pea cultivars were less competitive than leafy cultivars. Leafy pea cultivars had greater leaf area, plant height, and shoot DM attributes and greater root and shoot competitive abilities than semi-leafless pea cultivars. This shows that leafy pea cultivars not only had a greater leaf canopy but a more extensive root system than the semi-leafless pea cultivars. The difference in root competitive ability and shoot competitive ability between the two cultivars was the same, and this probably indicates that the difference in shoot morphology was reflected in root morphology.

Nitrogen uptake of pea cultivars varied between 144.5 and $288.1 \mathrm{~kg} \mathrm{ha}^{-1}$ depending on the seeding rate. The highest $\mathrm{N}$ uptake was obtained for 'Kirazli' at $125 \mathrm{~g}$ seed $\mathrm{m}^{-2}$ (Table 5). The $\mathrm{N}$ uptake was ranked as $125>100>150>75>175 \mathrm{~g}$ seed $\mathrm{m}^{2}$ in both years (Table 6). It varied between $180.8 \mathrm{~kg} \mathrm{ha}^{-1}$ in 2010 and $240.7 \mathrm{~kg} \mathrm{ha}^{-1}$ in 2011 (Table 7).

Nitrogen is the most frequently required nutrient in a crop fertilization program. Applying $\mathrm{N}$ usually reduces $\mathrm{N}$ fixation, but starter $\mathrm{N}$ applied early and prior to the onset of $\mathrm{N}$ fixation has been recommended for field pea production when soil N content is low (Clayton et al., 1998). McKenzie et al. (2001) reported that there are no benefits from using starter $\mathrm{N}$ and, when there are, these are generally minimal. Deibert and Utter (2004) pointed out that the highest seed yields $\left(3.5 \mathrm{t} \mathrm{ha}^{-1}\right)$ were obtained when $135 \mathrm{~kg} \mathrm{ha}^{-1} \mathrm{~N}$ fertilizer was applied. Low $\mathrm{N}$ stress is one of the factors that most frequently occurs under high plant density and limits plant production. Low $\mathrm{N}$ availability in soils is an important yieldlimiting factor (Banziger and Lafitte, 1997). According to these results, the amount of applied N should be $210-280 \mathrm{~kg}$ $\mathrm{ha}^{-1}$ when the seeding rate is $125 \mathrm{~g}$ seed $\mathrm{m}^{2}$ as per the selected cultivars.

The $\mathrm{P}$ uptake of pea cultivars ranged from 17.3 to $31.0 \mathrm{~kg} \mathrm{ha}^{-1}$ depending on the seeding rate (Table 4). The cultivars were ranked as Kirazli $\left(26.1 \mathrm{~kg} \mathrm{ha}^{-1}\right)>$ Ürünlü $\left(22.7 \mathrm{~kg} \mathrm{ha}^{-1}\right)>\operatorname{Gölyazi}\left(22.5 \mathrm{~kg} \mathrm{ha}^{-1}\right)>$ Ulubatli $\left(20.6 \mathrm{~kg} \mathrm{ha}^{-1}\right)$ for P uptake.

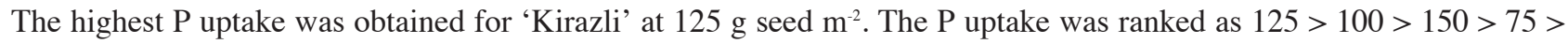
$175 \mathrm{~g}$ seed m $\mathrm{m}^{-2}$ in both years. It varied between $20.0 \mathrm{~kg} \mathrm{ha}^{-1}$ in 2010 and $25.9 \mathrm{~kg} \mathrm{ha}^{-1}$ in 2011.

Phosphorus is required for pea growth and $\mathrm{N}$ fixation. Sandaña and Pinochet (2014) reported that P uptake of pea ranged from 1.55 to $2.98 \mathrm{~kg} \mathrm{ha}^{-1}$. Therefore, applying 30-35 $\mathrm{kg} \mathrm{P}_{2} \mathrm{O}_{5} \mathrm{ha}^{-1}$ is sufficient to meet crop requirements (Lafond and Pageau, 2010). Other researchers have indicated that pea responds to high fertilization rates. Tawaha and Turk (2004)

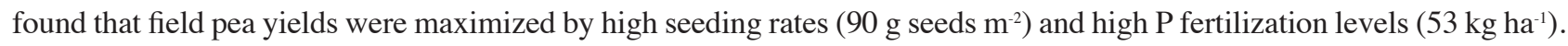


Phosphorus helps root development, flower initiation, and seed and fruit development; it has reduced disease incidence in some plants and has improved the quality of certain crops. According to these results, the amount of applied P should be 24.2-31.0 kg ha-1 when the seeding rate is $125 \mathrm{~g}^{-1} \mathrm{see} \mathrm{m}^{-2}$ as per the selected cultivars.

Table 5. Effects of different seeding rates on plant nutrient uptake of pea cultivars (Cultivar $\times$ Seeding rate).

\begin{tabular}{|c|c|c|c|c|c|c|}
\hline & \multicolumn{6}{|c|}{ Seeding rate } \\
\hline & $75 \mathrm{~g}$ & $100 \mathrm{~g}$ & $125 \mathrm{~g}$ & $150 \mathrm{~g}$ & $175 \mathrm{~g}$ & Mean \\
\hline Cultivars & \multicolumn{5}{|c|}{ Nitrogen, $\mathrm{kg} \mathrm{ha}^{-1}$} & \\
\hline Kirazli & $210.5 \mathrm{ef}$ & $256.7 b$ & $285.1 \mathrm{a}$ & $222.7 \mathrm{de}$ & $175.2 \mathrm{i}$ & $230.0 \mathrm{~A}$ \\
\hline Ulubatli & $144.5 \mathrm{j}$ & 184.0hi & $218.0 \mathrm{e}$ & 183.1hi & $150.2 \mathrm{j}$ & $176.0 \mathrm{C}$ \\
\hline Golyazi & $218.7 \mathrm{e}$ & $249.7 \mathrm{bc}$ & $288.1 \mathrm{a}$ & 208.7efg & $171.9 \mathrm{i}$ & $227.4 \mathrm{~A}$ \\
\hline Urunlu & 198.1fgh & $237.1 \mathrm{~cd}$ & $277.8 \mathrm{a}$ & $193.5 \mathrm{gh}$ & $140.6 \mathrm{j}$ & 209.4B \\
\hline Mean & 192.9D & 231.9B & $267.2 \mathrm{~A}$ & $202.0 \mathrm{C}$ & $159.5 \mathrm{E}$ & \\
\hline \multicolumn{7}{|c|}{ Phosphorus $\left(\mathrm{P}_{2} \mathrm{O}_{5}\right), \mathrm{kg} \mathrm{ha}^{-1}$} \\
\hline Kirazli & $22.1 \mathrm{e}-\mathrm{h}$ & $29.1 \mathrm{ab}$ & $31.0 \mathrm{a}$ & $26.7 b-d$ & $21.4 \mathrm{f}-\mathrm{j}$ & $26.1 \mathrm{~A}$ \\
\hline Ulubatli & $18.3 \mathrm{i}-\mathrm{k}$ & $21.8 \mathrm{f}-\mathrm{i}$ & $24.2 \mathrm{~d}-\mathrm{f}$ & $19.7 \mathrm{~g}-\mathrm{k}$ & $19.3 \mathrm{~h}-\mathrm{k}$ & $20.6 \mathrm{C}$ \\
\hline Golyazi & $23.1 \mathrm{e}-\mathrm{g}$ & $23.7 d-f$ & $25.5 \mathrm{c}-\mathrm{e}$ & $22.0 \mathrm{e}-\mathrm{h}$ & $18.2 \mathrm{jk}$ & $22.5 \mathrm{~B}$ \\
\hline Urunlu & $21.4 \mathrm{f}-\mathrm{j}$ & $23.1 \mathrm{e}-\mathrm{g}$ & $28.8 \mathrm{a}-\mathrm{c}$ & $23.1 \mathrm{a}$ & $17.3 \mathrm{k}$ & $22.7 \mathrm{~B}$ \\
\hline Mean & $21.2 \mathrm{C}$ & $24.4 \mathrm{~B}$ & $27.4 \mathrm{~A}$ & $22.9 \mathrm{BC}$ & 19.0D & \\
\hline \multicolumn{7}{|c|}{ Potassium $\left(\mathrm{K}_{2} \mathrm{O}\right), \mathrm{kg} \mathrm{ha}^{-1}$} \\
\hline Kirazli & 135.7 & 173.8 & 177.7 & 155.3 & 127.0 & $153.9 \mathrm{~A}$ \\
\hline Ulubatli & 95.0 & 139.8 & 134.9 & 107.1 & 108.9 & 117.2B \\
\hline Golyazi & 148.1 & 125.9 & 144.6 & 132.2 & 100.6 & $130.3 \mathrm{~B}$ \\
\hline Urunlu & 151.3 & 154.3 & 183.7 & 155.6 & 997.0 & $148.9 \mathrm{~A}$ \\
\hline Mean & $132.5 \mathrm{C}$ & $148.5 \mathrm{AB}$ & $160.2 \mathrm{~A}$ & $137.6 \mathrm{BC}$ & 109.0D & \\
\hline \multicolumn{7}{|c|}{ Calcium, kg ha-1 } \\
\hline Kirazli & $71.8 \mathrm{~cd}$ & $75.9 a-c$ & $85.5 \mathrm{a}$ & $74.3 \mathrm{a}-\mathrm{c}$ & $56.6 \mathrm{ef}$ & $72.8 \mathrm{~A}$ \\
\hline Ulubatli & $49.5 f g$ & $82.6 a-c$ & $61.9 \mathrm{de}$ & $60.4 \mathrm{ef}$ & $53.6 \mathrm{e}-\mathrm{g}$ & $61.6 \mathrm{~B}$ \\
\hline Golyazi & $76.8 \mathrm{a}-\mathrm{c}$ & $77.6 a-c$ & $83.9 \mathrm{ab}$ & $73.2 \mathrm{bc}$ & $54.9 \mathrm{e}-\mathrm{g}$ & $73.3 \mathrm{~A}$ \\
\hline Urunlu & 61.6de & $74.3 \mathrm{a}-\mathrm{c}$ & $78.9 a-c$ & $58.9 \mathrm{ef}$ & $43.8 \mathrm{~g}$ & $63.5 \mathrm{~B}$ \\
\hline Mean & $64.9 \mathrm{~B}$ & $77.6 \mathrm{~A}$ & $77.5 \mathrm{~A}$ & $66.7 \mathrm{~B}$ & $52.2 \mathrm{C}$ & \\
\hline \multicolumn{7}{|c|}{ Magnesium, kg ha-1 } \\
\hline Kirazli & $14.3 \mathrm{~d}-\mathrm{g}$ & $17.2 \mathrm{ab}$ & $18.2 \mathrm{a}$ & $15.8 b-d$ & $12.7 \mathrm{~g}-\mathrm{i}$ & $15.6 \mathrm{~A}$ \\
\hline Ulubatli & $10.6 \mathrm{jk}$ & $15.9 b-d$ & $13.7 \mathrm{e}-\mathrm{h}$ & $12.7 \mathrm{~g}-\mathrm{i}$ & $11.9 \mathrm{~h}-\mathrm{j}$ & $13.0 \mathrm{C}$ \\
\hline Golyazi & $15.3 \mathrm{~b}-\mathrm{f}$ & $15.1 \mathrm{c}-\mathrm{f}$ & $15.5 b-e$ & $14.0 \mathrm{~d}-\mathrm{g}$ & $11.0 \mathrm{i}-\mathrm{k}$ & $14.2 \mathrm{~B}$ \\
\hline Urunlu & $12.8 \mathrm{~g}-\mathrm{i}$ & $14.7 \mathrm{~d}-\mathrm{g}$ & $16.8 \mathrm{a}-\mathrm{c}$ & $13.3 \mathrm{f}-\mathrm{h}$ & $9.6 \mathrm{k}$ & $13.4 \mathrm{BC}$ \\
\hline Mean & $13.3 \mathrm{~B}$ & $15.7 \mathrm{~A}$ & $16.1 \mathrm{~A}$ & $14.0 \mathrm{~B}$ & $11.3 \mathrm{C}$ & \\
\hline \multicolumn{7}{|c|}{ Sodium, $\mathrm{kg} \mathrm{ha}^{-1}$} \\
\hline Kirazli & $4.909 \mathrm{~d}-\mathrm{f}$ & $6.535 \mathrm{ab}$ & $6.500 \mathrm{ab}$ & $5.574 \mathrm{~cd}$ & $4.932 c-f$ & $5.690 \mathrm{~A}$ \\
\hline Ulubatli & $3.828 \mathrm{~g}$ & $5.163 \mathrm{c}-\mathrm{e}$ & $5.221 \mathrm{c}-\mathrm{e}$ & $5.137 \mathrm{c}-\mathrm{e}$ & $4.180 \mathrm{fg}$ & $4.706 \mathrm{C}$ \\
\hline Golyazi & $5.368 \mathrm{c}-\mathrm{e}$ & $4.844 \mathrm{~d}-\mathrm{f}$ & $5.748 \mathrm{bc}$ & $4.726 \mathrm{ef}$ & $3.772 \mathrm{~g}$ & $4.892 \mathrm{BC}$ \\
\hline Urunlu & $5.181 \mathrm{c}-\mathrm{e}$ & $5.270 \mathrm{c}-\mathrm{e}$ & $6.618 \mathrm{a}$ & $5.069 \mathrm{c}-\mathrm{e}$ & $3.570 \mathrm{~g}$ & $5.142 B$ \\
\hline Mean & $4.821 \mathrm{C}$ & $5.453 \mathrm{~B}$ & $6.022 \mathrm{~A}$ & $5.127 \mathrm{BC}$ & 4.114D & \\
\hline \multicolumn{7}{|c|}{ Iron, $\mathrm{g} \mathrm{ha}^{-1}$} \\
\hline Kirazli & $451.1 \mathrm{bc}$ & $659.5 b c$ & $725.6 \mathrm{abc}$ & $574.5 \mathrm{bc}$ & $432.0 \mathrm{bc}$ & $568.6 \mathrm{~A}$ \\
\hline Ulubatli & $393.6 b c$ & $643.1 \mathrm{bc}$ & $516.4 b c$ & $436.8 b c$ & $499.9 b c$ & $498.0 \mathrm{~A}$ \\
\hline Golyazi & $672.1 b c$ & $1088.2 \mathrm{a}$ & $608.6 b c$ & $501.1 b c$ & $401.4 \mathrm{bc}$ & $654.3 \mathrm{~A}$ \\
\hline Urunlu & $561.8 b c$ & $797.3 \mathrm{ab}$ & $655.0 \mathrm{bc}$ & $449.6 b c$ & $358.5 \mathrm{c}$ & $564.4 \mathrm{~A}$ \\
\hline Mean & $519.7 \mathrm{~B}$ & $797.0 \mathrm{~A}$ & $626.4 \mathrm{AB}$ & $490.5 \mathrm{~B}$ & $422.9 \mathrm{~B}$ & \\
\hline \multicolumn{7}{|c|}{ Copper, $\mathrm{g} \mathrm{ha}^{-1}$} \\
\hline Kirazli & $39.2 d-f$ & $49.4 a-c$ & $50.2 \mathrm{ab}$ & $43.9 b-d$ & $37.6 \mathrm{~d}-\mathrm{g}$ & $44.1 \mathrm{~A}$ \\
\hline Ulubatli & $31.4 \mathrm{gh}$ & $40.9 \mathrm{~d}-\mathrm{f}$ & $41.5 \mathrm{~d}-\mathrm{f}$ & $36.2 \mathrm{e}-\mathrm{h}$ & $34.6 \mathrm{f}-\mathrm{g}$ & 36.9B \\
\hline Golyazi & $43.9 b-d$ & $43.5 b-d$ & 43.6b-d & $43.0 \mathrm{c}-\mathrm{e}$ & $32.0 \mathrm{~g}-\mathrm{h}$ & $41.2 \mathrm{~A}$ \\
\hline Urunlu & $39.7 d-f$ & $42.7 \mathrm{c}-\mathrm{e}$ & $52.8 \mathrm{a}$ & $40.9 d-f$ & $30.0 \mathrm{~h}$ & $41.2 \mathrm{~A}$ \\
\hline Mean & $38.5 \mathrm{C}$ & $44.1 \mathrm{AB}$ & $47.0 \mathrm{~A}$ & $41.0 \mathrm{BC}$ & $33.6 \mathrm{D}$ & \\
\hline
\end{tabular}


Continuation Table 5.

\begin{tabular}{|c|c|c|c|c|c|c|}
\hline & \multicolumn{6}{|c|}{ Seeding rate } \\
\hline & $75 \mathrm{~g}$ & $100 \mathrm{~g}$ & $125 \mathrm{~g}$ & $150 \mathrm{~g}$ & $175 \mathrm{~g}$ & Mean \\
\hline Cultivars & \multicolumn{6}{|c|}{ Zinc, $\mathrm{g} \mathrm{ha}^{-1}$} \\
\hline Kirazli & 117.0 & 160.2 & 171.9 & 155.9 & 124.5 & $145.9 \mathrm{AB}$ \\
\hline Ulubatli & 111.6 & 128.7 & 154.8 & 137.8 & 118.9 & 130.4B \\
\hline Golyazi & 157.1 & 170.8 & 169.7 & 162.1 & 127.9 & $157.5 \mathrm{~A}$ \\
\hline Urunlu & 142.7 & 167.5 & 188.4 & 177.0 & 122.4 & $159.6 \mathrm{~A}$ \\
\hline Mean & $132.1 \mathrm{~B}$ & $156.8 \mathrm{~A}$ & $171.2 \mathrm{~A}$ & $158.2 \mathrm{~A}$ & $123.4 \mathrm{~B}$ & \\
\hline \multicolumn{7}{|c|}{ Manganese, $\mathrm{g} \mathrm{ha}^{-1}$} \\
\hline Kirazli & 240.2 & 304.3 & 329.6 & 275.5 & 229.5 & $275.8 \mathrm{~B}$ \\
\hline Ulubatli & 224.1 & 302.9 & 238.3 & 240.3 & 212.4 & $243.6 \mathrm{C}$ \\
\hline Golyazi & 304.7 & 280.2 & 305.5 & 248.2 & 194.9 & 266.7BC \\
\hline Urunlu & 288.3 & 426.2 & 389.9 & 266.9 & 240.1 & $322.3 \mathrm{~A}$ \\
\hline Mean & $264.3 \mathrm{~B}$ & $328.4 \mathrm{~A}$ & $315.8 \mathrm{~A}$ & $257.7 \mathrm{~B}$ & $219.2 \mathrm{C}$ & \\
\hline
\end{tabular}

Uppercase letters in the same row and lowercase letters in the same column indicate differences $(P<0.05)$ according to Duncan's test.

Phosphorus fertilization should be based on soil analysis. Higher $\mathrm{P}$ fertilization rates are recommended if $\mathrm{P}$ is deficient in the soil. In soils with optimum to high P levels, farmers should add the amount of P removed by the pea crop to maintain the soil with an adequate $\mathrm{P}$ level. The $\mathrm{P}$ deficiency causes a purple color in leaves. Moreover, in soils with moderate plant available $\mathrm{K}$ status, applying $50 \mathrm{~kg} \mathrm{ha}^{-1}$ is sufficient to meet crop requirements and maintain soil fertility (Lafond and Pageau, 2010).

Potassium has many functions in plant growth. It is essential for photosynthesis, activates enzymes to metabolize carbohydrates to manufacture amino acids and proteins, facilitates cell division and growth by helping to move starches and sugars between plant parts, adds stalk and stem stiffness, increases disease resistance, increases drought tolerance. It also regulates the opening and closing of stomata, gives plumpness to the grain and seed, improves firmness, texture, size and color of fruit crops, and increases the oil content of oil crops (Romheld and Kirkby, 2010).

The K uptake of pea cultivars varied between 95.0 and $183.7 \mathrm{~kg} \mathrm{ha}^{-1}$ depending on the seeding rate. The cultivars were ranked as Kirazli $\left(153.9 \mathrm{~kg} \mathrm{ha}^{-1}\right)>$ Ürünlü $\left(148.9 \mathrm{~kg} \mathrm{ha}^{-1}\right)>\operatorname{Gölyazi}\left(130.3 \mathrm{~kg} \mathrm{ha}^{-1}\right)>$ Ulubatli $\left(117.2 \mathrm{~kg} \mathrm{ha}^{-1}\right)$ for K uptake.

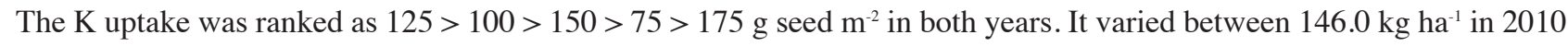
and $129.1 \mathrm{~kg} \mathrm{ha}^{-1}$ in 2011.

Potassium stimulates the processes of protein synthesis and the linking of amino acids in simple proteins. In soils with a low available $\mathrm{K}$ concentration, the recommended mean rate of this component should be increased by $20 \%$ to $30 \%$. The recommended $\mathrm{K}$ rate is $80-100 \mathrm{~kg} \mathrm{ha}^{-1}$ for a mean pea seed yield of 3-4 $\mathrm{Mg} \mathrm{ha}^{-1}$ (Czuba, 2001).

Table 6. Effects of different seeding rates on plant nutrient uptake of pea cultivars (Cultivar $\times$ Year).

\begin{tabular}{|c|c|c|c|c|c|c|c|c|c|c|}
\hline Cultivars & $\mathrm{N}$ & $\mathrm{P}$ & $\mathrm{K}$ & $\mathrm{Ca}$ & $\mathrm{Mg}$ & $\mathrm{Na}$ & $\mathrm{Fe}$ & $\mathrm{Cu}$ & $\mathrm{Zn}$ & $\mathrm{Mn}$ \\
\hline & \multicolumn{5}{|c|}{$\longrightarrow \mathrm{kg} \mathrm{ha}^{-1} \longrightarrow$} & \multicolumn{5}{|c|}{$\mathrm{g} \mathrm{ha}^{-1}$} \\
\hline \multicolumn{11}{|c|}{2010} \\
\hline Kirazli & 200.9 & 24.0 & $171.7 \mathrm{a}$ & $79.1 \mathrm{a}$ & 15.4 & 4.697 & 581.1 & 43.7 & 119.5 & 299.9 \\
\hline Ulubatli & 143.3 & 18.0 & $125.8 \mathrm{~cd}$ & $65.1 b c$ & 12.3 & 3.705 & 442.2 & 34.1 & 110.3 & 242.3 \\
\hline Golyazi & 198.2 & 18.9 & $136.1 b c$ & $77.5 \mathrm{a}$ & 12.7 & 4.029 & 548.4 & 34.6 & 127.0 & 271.9 \\
\hline Urunlu & 180.8 & 19.3 & $150.4 \mathrm{~b}$ & $66.2 \mathrm{bc}$ & 12.0 & 4.138 & 523.5 & 35.9 & 131.2 & 307.3 \\
\hline Mean & $180.8 \mathrm{~B}$ & $20.0 \mathrm{~B}$ & $146.0 \mathrm{~A}$ & $72.0 \mathrm{~A}$ & $13.1 \mathrm{~B}$ & 4.143B & $523.8 \mathrm{~B}$ & 37.1 & $122.0 \mathrm{~B}$ & $280.4 \mathrm{~A}$ \\
\hline \multicolumn{11}{|c|}{2011} \\
\hline Kirazli & 259.2 & 28.1 & $136.0 \mathrm{bc}$ & $66.5 \mathrm{bc}$ & 15.9 & 6.683 & 556.0 & 44.4 & 172.2 & 251.7 \\
\hline Ulubatli & 208.7 & 23.3 & $108.5 \mathrm{~d}$ & $58.0 \mathrm{~d}$ & 13.6 & 5.706 & 553.8 & 39.7 & 150.4 & 244.8 \\
\hline Golyazi & 256.7 & 26.0 & $124.4 \mathrm{~cd}$ & $69.1 \mathrm{~b}$ & 15.7 & 5.754 & 760.1 & 47.8 & 188.0 & 261.5 \\
\hline Urunlu & 238.0 & 26.1 & $147.5 b$ & $60.8 \mathrm{~cd}$ & 14.9 & 6.145 & 605.3 & 46.6 & 188.1 & 337.3 \\
\hline Mean & $24.07 \mathrm{~A}$ & $25.9 \mathrm{~A}$ & 129.1B & $63.6 \mathrm{~B}$ & $15.0 \mathrm{~A}$ & $6.072 \mathrm{~A}$ & $618.8 \mathrm{~A}$ & 44.6 & $174.7 \mathrm{~A}$ & $273.8 \mathrm{~A}$ \\
\hline
\end{tabular}

Uppercase letters in the same row and lowercase letters in the same column indicate differences $(P<0.05)$ according to Duncan's test. 
Table 7. Effects of different seeding rates on plant nutrient uptake of pea cultivars (Seeding rate $\times$ Year).

\begin{tabular}{|c|c|c|c|c|c|c|c|c|c|c|}
\hline $\begin{array}{l}\text { Seeding rate } \\
\left(\mathrm{g} \mathrm{m}^{-2}\right)\end{array}$ & $\mathrm{N}$ & $\mathrm{P}$ & $\mathrm{K}$ & $\mathrm{Ca}$ & $\mathrm{Mg}$ & $\mathrm{Na}$ & $\mathrm{Fe}$ & $\mathrm{Cu}$ & $\mathrm{Zn}$ & $\mathrm{Mn}$ \\
\hline & \multicolumn{5}{|c|}{$\longrightarrow \mathrm{kg} \mathrm{ha}^{-1} \longrightarrow$} & \multicolumn{5}{|c|}{$\mathrm{g} \mathrm{ha}^{-1}$} \\
\hline \multicolumn{11}{|c|}{2010} \\
\hline 75 & $176.5 \mathrm{f}$ & $19.9 \mathrm{de}$ & 146.6 & 72.4 & $13.2 \mathrm{~d}$ & 4.072 & $552.1 \mathrm{~b}$ & $36.8 \mathrm{de}$ & 116.6de & $284.8 \mathrm{bc}$ \\
\hline 100 & 199.0de & $21.9 \mathrm{~cd}$ & 162.7 & 82.9 & $14.9 \mathrm{c}$ & 4.583 & $602.0 \mathrm{~b}$ & $40.7 \mathrm{~cd}$ & $135.9 \mathrm{~cd}$ & $335.6 \mathrm{a}$ \\
\hline 125 & $229.5 \mathrm{c}$ & $23.8 \mathrm{c}$ & 171.8 & 83.1 & $15.0 \mathrm{c}$ & 4.844 & $613.5 b$ & $42.8 \mathrm{bc}$ & $133.0 \mathrm{~cd}$ & $319.1 \mathrm{ab}$ \\
\hline 150 & $169.1 \mathrm{f}$ & $18.7 \mathrm{e}$ & 133.1 & 68.6 & $12.3 \mathrm{~d}$ & 3.896 & $473.2 \mathrm{~b}$ & $34.2 \mathrm{ef}$ & $123.3 \mathrm{c}-\mathrm{e}$ & $243.9 \mathrm{de}$ \\
\hline 175 & $129.8 \mathrm{f}$ & $16.0 \mathrm{f}$ & 115.8 & 52.9 & $10.0 \mathrm{e}$ & 3.318 & $378.2 \mathrm{~b}$ & $30.9 \mathrm{f}$ & $101.3 \mathrm{e}$ & $218.4 \mathrm{e}$ \\
\hline Mean & 180.8 & 20.0 & 146.0 & 72.0 & 13.1 & 4.143 & 523.8 & 37.1 & 122.0 & 280.4 \\
\hline \multicolumn{11}{|c|}{2011} \\
\hline K75 & $209.4 \mathrm{~d}$ & $22.5 \mathrm{c}$ & 118.4 & 57.5 & $13.3 \mathrm{~d}$ & 5.571 & $487.2 \mathrm{~b}$ & $40.3 \mathrm{~cd}$ & $147.6 \mathrm{c}$ & $243.8 \mathrm{de}$ \\
\hline 100 & $264.8 \mathrm{~b}$ & $26.9 \mathrm{~b}$ & 134.3 & 72.3 & $16.5 \mathrm{ab}$ & 6.323 & $992.0 \mathrm{a}$ & $47.6 \mathrm{ab}$ & $177.8 \mathrm{~b}$ & $321.2 \mathrm{ab}$ \\
\hline 125 & $305.0 \mathrm{a}$ & $30.9 \mathrm{a}$ & 148.7 & 72.0 & $17.1 \mathrm{a}$ & 7.199 & $639.3 b$ & $51.2 \mathrm{a}$ & $209.5 \mathrm{a}$ & $312.5 \mathrm{ab}$ \\
\hline 150 & $234.9 \mathrm{c}$ & $27.0 \mathrm{~b}$ & 142.0 & 64.8 & $15.6 b c$ & 6.357 & $507.9 \mathrm{~b}$ & $47.8 \mathrm{a}$ & 193.1ab & $271.5 \mathrm{~cd}$ \\
\hline 175 & $189.1 \mathrm{e}$ & $22.1 \mathrm{~cd}$ & 102.3 & 51.6 & $12.7 \mathrm{~d}$ & 4.909 & $467.6 \mathrm{~b}$ & 36.3de & $145.6 \mathrm{c}$ & $220.0 \mathrm{e}$ \\
\hline Mean & 240.7 & 25.9 & 129.1 & 63.6 & 15.0 & 6.072 & 618.8 & 44.6 & 174.7 & 273.8 \\
\hline
\end{tabular}

Uppercase letters in the same row and lowercase letters in the same column indicate differences $(P<0.05)$ according to Duncan's test.

In a 2-yr field study conducted in Melfort, Saskatchewan, nutrient uptake in the pea seed at harvest was estimated as $132 \mathrm{~kg} \mathrm{~N} \mathrm{ha}^{-1}, 14 \mathrm{~kg} \mathrm{P} \mathrm{ha}^{-1}$, and $40 \mathrm{~kg} \mathrm{~K} \mathrm{ha}^{-1}$ in the first year, while the nutrient uptake was $79 \mathrm{~kg} \mathrm{~N} \mathrm{ha}^{-1}, 9 \mathrm{~kg} \mathrm{P} \mathrm{ha}^{-1}$, and $24 \mathrm{~kg} \mathrm{~K} \mathrm{ha}^{-1}$ in the second year; this wide temporal variation in nutrient uptake in the second year is attributed to lower seed yield and harvest indexes due to hail damage (Malhi et al., 2007). A study in North Dakota reported nutrient requirements of field pea as $0.034 \mathrm{~kg} \mathrm{~N}, 0.0041 \mathrm{~kg} \mathrm{P}$, and $0.0115 \mathrm{~kg} \mathrm{~K}$ per kilogram seed yield at harvest, indicating that tillage practices, fertilization, and their interaction with the climate influence biomass accumulation, seed yield, and nutrient uptake in the field pea production systems (Deibert and Utter, 2004).

Calcium uptake of pea cultivars varied between 43.8 and $85.5 \mathrm{~kg} \mathrm{ha}^{-1}$ depending on seeding rate. The highest Ca uptake was obtained for 'Kirazli' at $125 \mathrm{~g}$ seed $\mathrm{m}^{-2}$. The Ca uptake was ranked as $125>100>150>75>175 \mathrm{~g} \mathrm{seed} \mathrm{m}^{-2}$ in both experimental years. It ranged from $72.0 \mathrm{~kg} \mathrm{ha}^{-1}$ in 2010 to $63.6 \mathrm{~kg} \mathrm{ha}^{-1}$ in 2011 . Candráková et al. (2014) reported that the Ca uptake of pea (seed + biomass) was $41.09 \mathrm{~kg} \mathrm{ha}^{-1}$ under the conditions of a warm and moderately arid continental climate with an average annual temperature of $9.07^{\circ} \mathrm{C}$ and average annual precipitation of $561 \mathrm{~mm}$. According to Fecenko and Lozek (2000), pea seeds using normal nutrients extract $25 \mathrm{~kg} \mathrm{Ca} \mathrm{t}^{-1}$ from the crop.

Calcium is a component of the cell walls and participates in producing new growing points and root tips. It provides elasticity and expansion to the cell walls, which keeps growing points from becoming rigid and brittle. It is immobile in plants and remains in the older tissue throughout the growing season. It acts as a base for neutralizing organic acids generated during the growing process and helps carbohydrate translocation and $\mathrm{N}$ absorption (Hepler, 2005).

Magnesium uptake of pea cultivars varied between 9.6 and $18.2 \mathrm{~kg} \mathrm{ha}^{-1}$ depending on the seeding rate. The cultivars were ranked for $\mathrm{Mg}$ as Kirazli $\left(15.6 \mathrm{~kg} \mathrm{ha}^{-1}\right)>\operatorname{Gölyazi}\left(14.2 \mathrm{~kg} \mathrm{ha}^{-1}\right)>$ Ürünlü $\left(13.4 \mathrm{~kg} \mathrm{ha}^{-1}\right)>$ Ulubatli $\left(13.0 \mathrm{~kg} \mathrm{ha}^{-1}\right)$. The

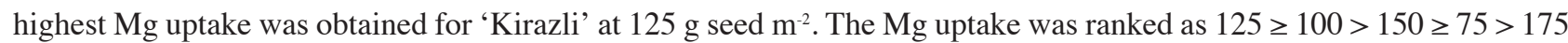
$\mathrm{g}$ seed $\mathrm{m}^{-2}$ in 2010 and $125>100>150>75 \geq 175 \mathrm{~g}$ seed $\mathrm{m}^{-2}$ in 2011. Candráková et al. (2014) reported that Mg uptake of pea (seed + biomass) was $13.91 \mathrm{~kg} \mathrm{ha}^{-1}$ under the conditions of a warm and moderately arid continental climate with an average annual temperature of $9.07^{\circ} \mathrm{C}$ average and annual precipitation of $561 \mathrm{~mm}$. According to Fecenko and Lozek (2000), pea seeds using normal nutrients extract $3.6 \mathrm{~kg} \mathrm{Mg} \mathrm{t}^{-1}$ from the crop.

Magnesium is a component of the chlorophyll molecule, which is the driving force of photosynthesis. It is essential for carbohydrate (sugars) metabolism, and is an enzyme activator in nucleic acid (DNA and RNA) synthesis. It regulates the uptake of other essential elements, serves as a carrier of phosphate compounds throughout the plant, facilitates carbohydrate (sugars and starches) translocation, and enhances oil and fat production (Guo et al., 2016).

Sodium uptake of pea cultivars ranged from 3.570 to $6.535 \mathrm{~kg} \mathrm{ha}^{-1}$ depending on the seeding rate. The cultivars were ranked as Kirazli $\left(5.690 \mathrm{~kg} \mathrm{ha}^{-1}\right)>$ Ürünlü $\left(5.142 \mathrm{~kg} \mathrm{ha}^{-1}\right)>$ Gölyazi $\left(4.892 \mathrm{~kg} \mathrm{ha}^{-1}\right)>$ Ulubatli $\left(4.706 \mathrm{~kg} \mathrm{ha}^{-1}\right)$. The 
highest Na uptake was obtained for 'Kirazli' at $100 \mathrm{~g}$ seed $\mathrm{m}^{-2}$. The Na uptake was ranked as $125>100>150>75>$ $175 \mathrm{~g} \mathrm{seed}^{-2}$ in both years.

Micronutrients are indispensable for plant growth and play an important role in balanced crop nutrition. Some important micronutrients include $\mathrm{Cu}, \mathrm{Fe}, \mathrm{Mn}$, and $\mathrm{Zn}$. They are as important to plant nutrition just as $\mathrm{N}, \mathrm{P}, \mathrm{K}, \mathrm{Ca}, \mathrm{Mg}, \mathrm{Na}, \mathrm{Fe}, \mathrm{Cu}$, $\mathrm{Zn}$, and $\mathrm{Mn}$. A lack of any one of the micronutrients in the soil can limit plant growth, even if the other soil nutrients are in sufficient amounts.

Iron is important for crop growth and food production. Plant Fe uptake is through the ferrous $\left(\mathrm{Fe}^{2+}\right)$ cation. Iron is a component of many enzymes associated with energy transfer, $\mathrm{N}$ reduction and fixation, and lignin formation (Rout and Sahoo, 2015). The Fe uptake of pea cultivars varied between 358.5 and $1088.2 \mathrm{~g} \mathrm{ha}^{-1}$ depending on the seeding rate. The highest Fe uptake was obtained for 'Gölyazi' at $100 \mathrm{~g}$ seed m $\mathrm{m}^{-2}$. It was $523.8 \mathrm{~g} \mathrm{ha}^{-1}$ in 2010 and $618.8 \mathrm{~g} \mathrm{ha}^{-1}$ in 2011.

Copper uptake of pea cultivars varied between 30.0 and $52.8 \mathrm{~g} \mathrm{ha}^{-1}$ depending on the seeding rate. Cultivars were ranked as Kirazli $\left(44.1 \mathrm{~g} \mathrm{ha}^{-1}\right)>$ Ürünlü $\left(41.2 \mathrm{~g} \mathrm{ha}^{-1}\right)=$ Gölyazi $\left(41.2 \mathrm{~g} \mathrm{ha}^{-1}\right)>$ Ulubatli $\left(36.9 \mathrm{~g} \mathrm{ha}^{-1}\right)$. The highest Cu uptake was obtained for 'Kirazli' and 'Urunlu' at $125 \mathrm{~g}$ seed $\mathrm{m}^{-2}$. The Cu uptake was ranked as $125>100>150>75>175 \mathrm{~g}$ seed $\mathrm{m}^{-2}$ in both years. Copper activates enzymes and catalyzes reactions in several plant growth processes (Ayala and Sandmann, 1988; Adhikari et al., 2016).

Zinc uptake of pea cultivars ranged from 111.6 to $188.4 \mathrm{~g} \mathrm{ha}^{-1}$ depending on the seeding rate. The cultivars were ranked as Ürünlü > Gölyazi > Kirazli > Ulubatli in both experimental years. The highest Zn uptake was obtained for 'Urunlu' at $125 \mathrm{~g}$ seed m$^{-2}$. The $\mathrm{Cu}$ uptake was ranked as $100>125>150>75>175 \mathrm{~g} \mathrm{seed} \mathrm{m}^{-2}$ in 2010 and $125>150>100>175$ $>75 \mathrm{~g}$ seed $\mathrm{m}^{-2}$ in 2011 . Zinc is taken up by plants as the divalent $\mathrm{Zn}^{+2}$. It was one of the first micronutrients recognized as essential for plants and the one that most commonly limited yields. Although $\mathrm{Zn}$ is required only in small amounts, high yields are impossible without it.

Manganese uptake of pea cultivars varied between 194.9 and $426.2 \mathrm{~g} \mathrm{ha}^{-1}$ depending on the seeding rate. The $\mathrm{Mn}$

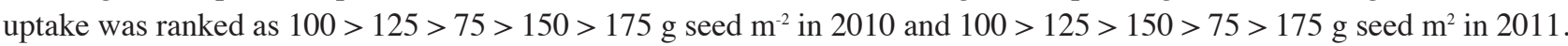

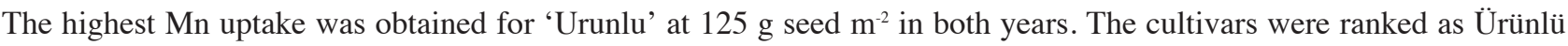
$>$ Gölyazi > Kirazli > Ulubatli in both yearsManganese primarily functions as part of plant enzyme systems. It activates several important metabolic reactions and plays a direct role in photosynthesis. Manganese accelerates germination and maturity while increasing P and Ca availability (Mousavi et al., 2011; Hafeez et al., 2013).

Harmankaya et al. (2010) reported that protein content and mineral composition of pea were significantly different among the 19 pea cultivars. In addition, researchers have mentioned that such variations in mineral content of pea samples might be due to their genetic origin. Several crops show genotypic differences in their competitive ability (McDonald et al., 2007). Results obtained in the study may be related to the genetic characteristics of pea cultivars. When choosing varieties for cultivation, it is important to consider their susceptibility to plant nutrient uptake and soil properties, climate adaptive capacity, and other agronomic features relevant to the growing region. Results obtained in this study were also comparable to the findings of other authors (Wang and Daun, 2004; Yoshida et al., 2007; Harmankaya et al., 2009).

\section{CONCLUSIONS}

There is considerable genetic variation in cultivated pea and a wide range of varieties is available, which have a broad spectrum of traits. When selecting a pea variety, one must consider crop use, region, sowing date, seeding rate, yield potential, frost and disease resistance or tolerance, ease of harvesting, and marketing options.

Seeding rate and pea cultivar are the most significant factors affecting yield parameters. Decreased yield is evident at seeding rates above or below the optimum rates. In this study, the effects of seeding rate on plant nutrient uptake (N, P, $\mathrm{K}, \mathrm{Ca}, \mathrm{Mg}, \mathrm{Na}, \mathrm{Fe}, \mathrm{Cu}, \mathrm{Zn}$, and $\mathrm{Mn}$ ) were determined in four forage pea cultivars under the conditions found in the Bursa Province, Turkey. Nitrogen, the most frequently obtained element, varied between 129.8 and $305.0 \mathrm{~kg} \mathrm{ha}^{-1}$ according to the pea cultivars and climatic conditions. In addition, $\mathrm{K}$ ranged from 108.5 to $177.0 \mathrm{~kg} \mathrm{ha}^{-1}, 58.0-79.1 \mathrm{~kg} \mathrm{Ca} \mathrm{ha}^{-1}$, and 18.0-28.1 kg P ha-1. Field pea cultivars contained various levels of microelements. According to this study and considering the cultivar $\times$ seeding rate interaction, 'Kirazli' and 'Golyazi' at $125 \mathrm{~g}$ seed $\mathrm{m}^{-2}$ were recommended for higher $\mathrm{N}$ uptake, 'Kirazli' for P uptake, and 'Kirazli' and 'Urunlu' for $\mathrm{K}$ and microelement uptake. The $\mathrm{Fe}, \mathrm{Cu}$, and $\mathrm{Zn}$ uptake of 'Golyazi' was higher than for the other cultivars. Plant density at approximately $125 \mathrm{~g} \mathrm{seed} \mathrm{m}^{-2}$ may be more suitable for pea grown 
under the study conditions, and increasing plant density does not significantly return increased product yield. These results revealed that field pea cultivars may provide a sufficient amount of plant nutrient elements to meet animal mineral requirements. We should pay attention to the mentioned issues when creating a fertilization program.

\section{ACKNOWLEDGEMENTS}

This research was financially supported by The Scientific Research Projects Unit of Bursa Uludag University (Project Nr 2010/15).

\section{REFERENCES}

Adhikari, T., Sarkar, D., Mashayekhi, H., and Xing, B. 2016. Growth and enzymatic activity of maize (Zea mays L.) plant: Solution culture test for copper dioxide nano particles. Journal of Plant Nutrition 39(1):99-115.

Ayala, B.M., and Sandmann, G. 1988. Activities of Cu-containing proteins in Cu-depleted pea leaves. Physiologia Plantarum 72:801-806.

Banziger, M., and Lafitte, H.R. 1997. Efficiency of secondary traits for improving maize for low-nitrogen target environments. Crop Science 37:1110-1117.

Candráková, E., Andrejcíková, M., and Hanácková, E. 2014. Yield formation of common peas and nutrient uptake. Research Journal of Agricultural Science 46(1):109-116.

Clayton, G., Rice, W., Blade, S., Grant, C., Harker, N., Johnston, A., et al. 1998. Minimizing risk and increasing yield stability in field pea production. In Proceedings from the $10^{\text {th }}$ Annual Meeting, Conference and Trade Show of the Saskatchewan Soil Conservation Association, Regina. 11-12 February. Saskatchewan Soil Conservation Association, Saskatoon, Saskatchewan, Canada.

Cupina, B., Krstic, D., Mikic, A., Eric, P., Vuckovic, S., and Pejic, B. 2010. The effect of field pea (Pisum sativum L.) companion crop management on red clover (Trifolium pratense L.) establishment and productivity. Turkish Journal of Agriculture and Forestry 34:275-283.

Czuba, R. 2001. Znaczenie potasu w polskim rolnictwie. International Potash Institute, Basel, Switzerland.

Dahmardeh, M., Ramroodi, M., and Valizadeh, J. 2010. Effect of plant density and cultivars on growth, yield and yield components of faba bean (Vicia faba L.) African Journal of Biotechnology 9(50):8643-8647.

Deibert, E.J., and Utter, R.A. 2004. Field pea growth and nutrient uptake: Response to tillage systems and nitrogen fertilizer applications. Communications in Soil Science and Plant Analysis 35:1141-1165.

Fecenko, J., and Lozek, O. 2000. Vyziva a hnojenie pol’nych plodín. 452 p. SPU v Nitre a Duslo, a.s., Sal'a.

Gan, Y.T., Miller, P.R., McConkey, B.G., Zentner, R.P., Liu, P.H., and McDonald, C.L. 2003. Optimum plant population density for chickpea and dry pea in a semiarid environment. Canadian Journal of Plant Science 83:1-9.

Guo, W., Nazimc, H., Lianga, Z., and Yanga, D. 2016. Magnesium deficiency in plants: An urgent problem. The Crop Journal 4:83-91.

Hafeez, B., Khanif, Y.M., and Saleem, M. 2013. Role of zinc in plant nutrition- A Review. American Journal of Experimental Agriculture 3(2):374-391.

Harmankaya, M., Karadas, S., Palta, C., and Ceyhan, E. 2009. Relationships between protein content and chemical compositions of local dry bean (Phaseolus vulgaris L.) genotypes grown in Turkey. Asian Journal of Chemistry 21(2):1535-1540.

Harmankaya, M., Ozcan, M.M., Karadas, S., and Ceyhan, E. 2010. Protein and mineral contents of pea (Pisum sativum L.) genotypes grown in Central Anatolian region of Turkey. South Western Journal of Horticulture, Biology and Environment 1(2):159-166.

Hepler, P.K. 2005. Calcium: A central regulator of plant growth and development. Plant Cell 17:2142-2155.

Kacar, B. 2014. Plant, soil and fertilizer analysis II. 423 p. Nobel Academic Publisher, Ankara, Turkey.

Karkanis, A., Ntatsi, G., Kontopoulou, C.K., Pristeri, A., Bilalis, D., and Savvas, D. 2016. Field pea in European cropping systems: Adaptability, biological nitrogen fixation and cultivation Practices. Not Bot Horti Agrobo 44(2):325-336.

Lafond, J., and Pageau, D. 2010. Phosphorus and potassium fertilization of dry pea. Canadian Journal of Plant Science 90(5):629-636.

Malhi, S.S., Johnston, A.M., Schoenau, J.J., Wang, Z.L., and Vera, C.L. 2007. Seasonal biomass accumulation and nutrient uptake of pea and lentil on a Black Chernozem Soil in Saskatchewan. Journal of Plant Nutrition 30(5):721-737.

McDonald, G.K., Hollaway, K.L., and McMurray, L. 2007. Increasing plant density improves weed competition in lentils (Lens culinaris). Australian Journal of Experimental Agriculture 47:48-56.

McKenzie, R.H., Middleton, A.B., Solberg, E.D., DeMulder, J., Flore, N., Clayton, G.W., et al. 2001. Response of pea to rhizobia inoculation and starter nitrogen in Alberta. Canadian Journal of Plant Science 81:637-643. 
Mousavi, S.R., Shahsavari, M., and Rezaei, M. 2011. General overview on manganese (Mn) importance for crops production. Australian Journal of Basic and Applied Sciences 5(9):1799-1803.

Olle, M. 2017. The effect of sowing rate and variety on the yield, protein content and height of field pea. International Journal of Food and Biosystems Engineering 3(1):11-18.

Özsoy, G., and Aksoy, E. 2004. Soils of the Uludag University Campus area, their genesis and classification. International Soil Congress (ISC) on Natural Resource Management for Sustainable Development, Erzurum, Turkey. 7-10 June. Abst. Book. p. 85. Soil Science Society of Turkey (SSST), Ankara, Turkey.

Özsoy, G., and Aksoy, E. 2007. Characterization, classification and agricultural usage of vertisols developed on neogen aged calcareous marl parent materials. Journal of Biological and Environmental Sciences 1(1):5-10.

Romheld, V., and Kirkby, E. 2010. Research on potassium in agriculture: Needs and prospects. Plant and Soil 335(1-2):155-180.

Rout, G.R., and Sahoo, S. 2015. Role of iron in plant growth and metabolism. Reviews in Agricultural Science 3:1-24.

Sandaña, P., and Pinochet, D. 2014. Grain yield and phosphorus use efficiency of wheat and pea in a high yielding environment. Journal of Soil Science and Plant Nutrition 14(4):973-986.

Semere, T., and Froud-Williams, R.J. 2001. The effect of pea cultivar and water stress on root and shoot competition between vegetative plants of maize and peas. Journal of Applied Ecology 38:137-145.

Siqueira, P.L., Silva, P.S.L., Silva, K.E.F., Oliveira, V.R., Dantas, I.M., and Oliveira, F.H.T. 2014. Soil fertility beneath the crown of tree species submitted to planting densities. Revista Brasileira de Engenharia Agrícola e Ambiental 18(9):914-919.

Spies, J.M., Warkentin, T., and Shirtliffe, S.J. 2010. Basal branching in field pea cultivars and yield-density relationships. Canadian Journal of Plant Science 90:679-690.

Tan, M., Koc, A., and Dumlu, G.Z. 2012. Morphological characteristics and seed yield of East Anatolian local forage pea (Pisum sativum ssp. arvense L.) ecotypes. Turkish Journal of Field Crops 17(1):24-30.

Tawaha, A.M., and Turk, M.A. 2004. Field pea seeding management for semi-arid Mediterranean conditions. Journal of Agronomy and Crop Science 190:86-92.

Thandiwe, N., and Rickertsen, J. 2011. Seeding rate and variety effects on yield, yield components, and economic return of field pea in the Northern Great Plains. Crop Management 10:1.

Türk, M., Albayrak, S., and Yüksel, O. 2011. Effect of seeding rate on the forage yields and quality in pea cultivars of differing leaf types. Turkish Journal of Field Crops 16(2):137-141.

Uzun, A., Asik, B.B., and Acikgöz, E. 2017. Effects of different seeding rates on forage yield and quality components in pea. Turkish Journal of Field Crops 22(1):126-133.

Wang, N., and Daun, J.K. 2004. Effect of variety and crude protein content on nutrients and certain antinutrients in field peas (Pisum sativum). Journal of the Science of Food and Agriculture 84:1021-1029.

Yoshida, H., Tomiyama, Y., Tanaka, M., and Mizushina, Y. 2007. Characteristic profiles of lipid classes, fatty acids and triacylglycerol molecular species of peas (Pisum sativum L.) European Journal of Lipid Science and Technology 109:600-607.

Zandi, P., Shirani-Rad, A.H., and Bazrkar-Khatibani, L. 2011. Agronomic study of fenugreek grown under different inrow spacing and nitrogen levels in a paddy field of Iran. American-Eurasian Journal of Agricultural and Environmental Sciences 10:544-550. 